\title{
Is it Possible to Re-use Mini-implants for Orthodontic Anchorage? Results of an In Vitro Study
}

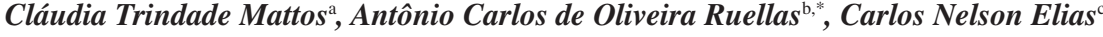 \\ ${ }^{a}$ Departamento de Ortodontia, Faculdade de Odontologia, Universidade Federal do Rio de \\ Janeiro - UFRJ, Av. Professor Rodolpho Paulo Rocco, 325, Ilha do Fundão, \\ CEP 21941-617, Rio de Janeiro, RJ, Brazil \\ ${ }^{\mathrm{b}}$ Departamento de Ortodontia, Faculdade de Odontologia, \\ Universidade Federal do Rio de Janeiro - UFRJ, Av. Professor Rodolpho Paulo Rocco, 325, \\ Ilha do Fundão, CEP 21941-617, Rio de Janeiro, RJ, Brazil \\ 'Departamento de Engenharia Mecânica e Ciência dos Materiais, Instituto Militar de \\ Engenharia, Laboratório de Biomateriais, Pr. General Tibúrcio, 80, Praia Vermelha, \\ CEP 22290-270, Rio de Janeiro, RJ, Brazil
}

Received: August 19, 2010; Revised: October 21, 2010

\begin{abstract}
The aim of this study was to compare the surface morphology and fracture torque resistance of as-received, sterilized and retrieved mini-implants in order to evaluate the fracture risks of re-using orthodontic mini-implants after sterilization. Thirty mini-implants retrieved from 19 patients, twenty as-received and twenty autoclaved miniimplants from the same manufacturer were used in this study. Ten samples from each group were analyzed by scanning electron microscopy and the remaining specimens were tested for their fracture torque resistance. Results were statistically analyzed using one-way ANOVA. No defects or corrosion could be identified in autoclaved and retrieved mini-implants, but a worn surface and scratch marks could be observed on the retrieved ones. A statistical significant difference in the fracture torque was observed between the as-received and the retrieved groups. Re-use of retrieved mini-implants should not be recommended because their torque resistance is reduced. As-received mini-implants submitted to autoclave sterilization can be recommended to be used clinically.
\end{abstract}

Keywords: implant fracture, re-use, Scanning Electron Microscopy (SEM), sterilization

\section{Introduction}

Mini-implants have been used for over a decade now since their introduction in Orthodontics as a skeletal anchorage system. They are an important aid in anchorage control and a determinant factor for success in orthodontic treatment. Their popularity is due especially to their simple placement with less traumatic surgery, less discomfort for patients, possibility of immediate loading and high versatility ${ }^{1-7}$.

Biomaterials may be used for temporary purposes in the body, like coverscrews for dental implants, wires or fracture fixation plates ${ }^{8}$. The mini-implants are included in this category, as they are removed once they are of no further assistance in the orthodontic treatment. After removal of temporary devices, the recovered devices are usually discarded. However, economic factors have caused some clinicians to re-use implants or other medical devices that are meant to be disposable, like pacemakers, intra-aortic balloons, haemodialysis membranes, coronary angioplasty catheters and orthodontic brackets and wires ${ }^{8-10}$. In case of re-use, it is mandatory to ensure the sterility and mechanical qualities of the device ${ }^{11}$. Not all implants can be re-used, but metal implants, like those made from titanium may be more amenable to re-use because they can be mechanically and chemically cleaned and re-sterilized with potentially little or no loss of form or function ${ }^{8}$.

One study investigated the characterization of retrieved orthodontic mini-implants by optical microscopy, Scanning Electron Microscopy (SEM), X-ray microtomography and X-ray microanalysis ${ }^{12}$, but no studies have assessed re-use of mini-implants. As previous use, cleaning and sterilization may contribute to changes in surface topography and mechanical resistance, it is important that these situations be studied to answer the question of whether miniimplants can be re-used or not.

Therefore, the objective of this study was to compare as-received, sterilized and retrieved mini-implants as to their surface topography and fracture torque resistance in order to evaluate the mechanical risks of re-using orthodontic mini-implants after sterilization.

\section{Materials and Methods}

All mini-implants in this study were of the same type and from the same manufacturer (SIN, São Paulo, Brazil), self-drilling, made from Ti6Al4V alloy and with a diameter of $1.4 \mathrm{~mm}$ and a thread length of $8 \mathrm{~mm}$.

Forty mini-implants from a single manufacturing lot were purchased from the manufacturer. Twenty mini-implants were submitted to a sterilization process in a dental steam autoclave (STERMAX, Pinhais, Brazil). The autoclave fulfilled the requirements of the British Stardards Institution ISO 9001:2000. Its efficacy was previously tested by a biological indicator test (Clean Test 10 , SIEGER, Campo Mourão, Brazil). Each mini-implant was inserted in an individual auto-sealing envelope (ZERMATT, São Paulo, Brazil) and all mini-implants were then submitted to one sterilizing cycle of 30 minutes at $121{ }^{\circ} \mathrm{C}$, according to the recommendations of the manufacturer of the autoclave. The other 20 mini-implants served as a control group and were analyzed and tested as received from the manufacturer. 
Thirty mini-implants were retrieved from 19 patients after successful service of 5 to 18 months (mean 9.73 months), with no signs of failure such as peri-implant soft-tissue inflammation and implant mobility or premature loss. Written informed consent was obtained from all patients and the research was approved by a local Ethics Committee. The mini-implants were removed by application of counterclockwise torquing load with a specially designed screwdriver provided by the manufacturer. After removal, each mini-implant was stored, completely immersed in distilled water, in a sterile pot used for laboratorial exams.

The mini-implants were divided into three groups, according to their condition - as-received (control), autoclaved or retrieved. The characteristics of each group are described in Table 1.

Before being analyzed in the scanning electron microscope, the specimens from the retrieved group were submitted to a cleaning cycle of 30 minutes in an ultrasonic washer (Maxiclean 1400A, Unique, Indaiatuba, Brazil), completely immersed in enzymatic detergent (Endozyme, DFL, Rio de Janeiro, Brazil) so that organic debris could be removed and the surface topography of the mini-implants could be fully observed in the microscope.

The mini-implants were mounted on aluminum supporting discs using double carbon sided tape. Surface effects were analyzed by SEM at $20 \mathrm{kV}$ (JEOL LSM-5800, Tokyo, Japan). Each mini-implant was examined for signs of corrosion, changes in morphology and surface alterations at various magnifications. Special attention was given to the thread and sharp tip of mini-implants, observed at $100 \times, 250 \times$ and 500× magnifications. Digital images were acquired by SEM.

Fracture torque testing was carried out by inserting a mini-implant into $9 \mathrm{~mm}$ thick cortical bone obtained from the femur of country pigs. Four bone segments were prepared so as to fit the device used in the test. A raster of 10 implantation sites with a minimum distance of $4 \mathrm{~mm}$ from each other was marked on the bone segments and pilot drilling was done using a $1.0 \mathrm{~mm}$ diameter drill (SIN, São Paulo, Brazil). A digital torque gauge (TQ-680, Instrutherm, São Paulo, Brazil) was perpendicularly positioned to the bone surface by using a device specially prepared for such a purpose (Figure 1). This torque test setup methodology was used before by Pithon et al. ${ }^{13}$, thus allowing the mini-implants to be correctly inserted manually and the fracture torque to be measured. Also, this device allowed the digital torque gauge to approximate as the mini-implant was inserted into the bone cortical in addition to avoid lateral movements, which might result in bascular fracture. The maximum torque reached before fracture of the mini-implant was recorded in $\mathrm{Ncm}$.

Data from all groups were analyzed with the SPSS statistical software for Windows (vs. 14.0, Chicago, IL, USA). Statistical descriptive analysis, including mean, standard deviation, median, minimum and maximum values, was calculated. The normality and equality of variance of data were checked by the KolmogorovSmirnov test. Results were statistically analyzed using one-way ANOVA with the Tukey HSD post-hoc test to detect differences among groups. A $P$-value of $<0.05$ was accepted as statistically significant.

Table 1. Characterization of mini-implants groups tested.

\begin{tabular}{cccccc}
\hline Group & Type & SEM & $\begin{array}{c}\text { Fracture } \\
\text { torque test }\end{array}$ & $\begin{array}{c}\text { Diameter } \\
(\mathrm{mm})\end{array}$ & $\begin{array}{c}\text { Thread length } \\
(\mathrm{mm})\end{array}$ \\
\hline $\mathrm{A}$ & $\begin{array}{c}\text { Autoclaved } \\
\text { Control }\end{array}$ & 10 & 10 & 1.4 & 8 \\
$\mathrm{C}$ & $\begin{array}{c}\text { Cos-received) } \\
\text { (as }\end{array}$ & 10 & 10 & 1.4 & 8 \\
$\mathrm{R}$ & Retrieved & 10 & 20 & 1.4 & 8 \\
\hline
\end{tabular}

\section{Results}

Figure 2 shows the surface morphology at 100x magnification of the tip of a retrieved mini-implant showing a less sharp tip and smooth surface.

Figure 3 shows the surface morphology at $250 \times$ magnification of an as-received, an autoclaved and a retrieved mini-implant visualized in SEM. The photomicrographs clearly show grooves due to the machining process. No defects in the form of pores or cracks and no image suggestive of corrosion could be identified in the autoclaved and retrieved mini-implants as compared to the as-received one. The retrieved mini-implant shows a smoother surface on the threads and scratch marks on the tip, when compared to the as-received and the autoclaved mini-implants.

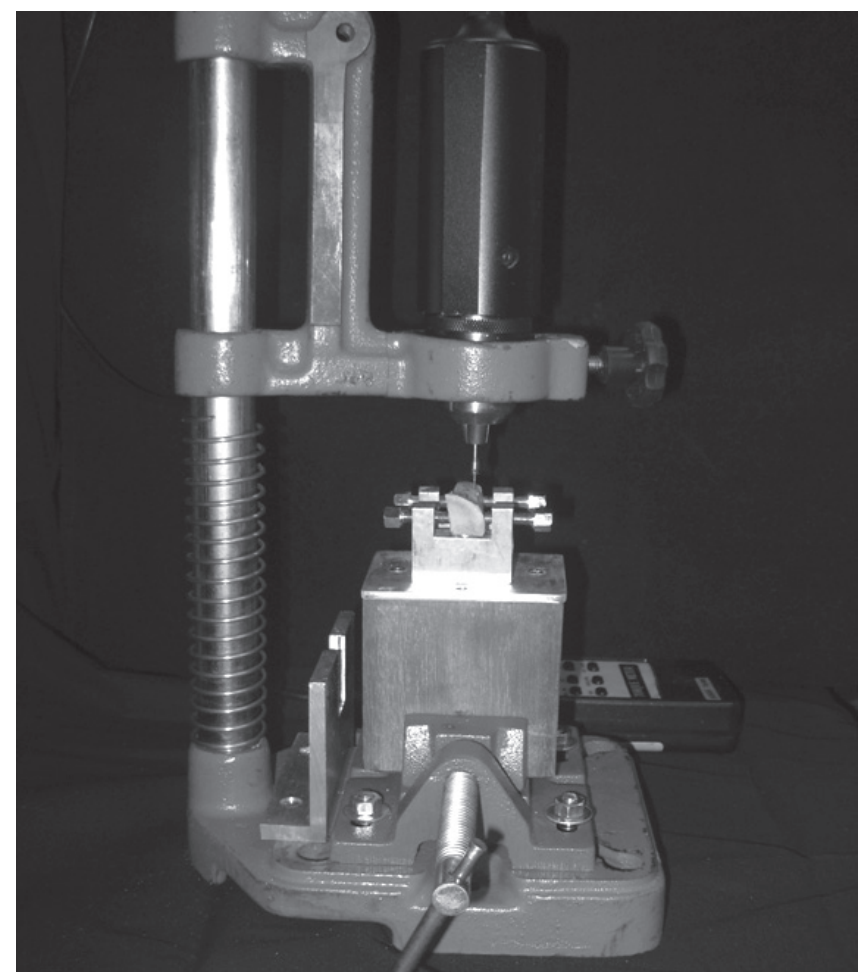

Figure 1. Photograph of the fracture test device showing a digital torque gauge attached to it and bone segment positioned.

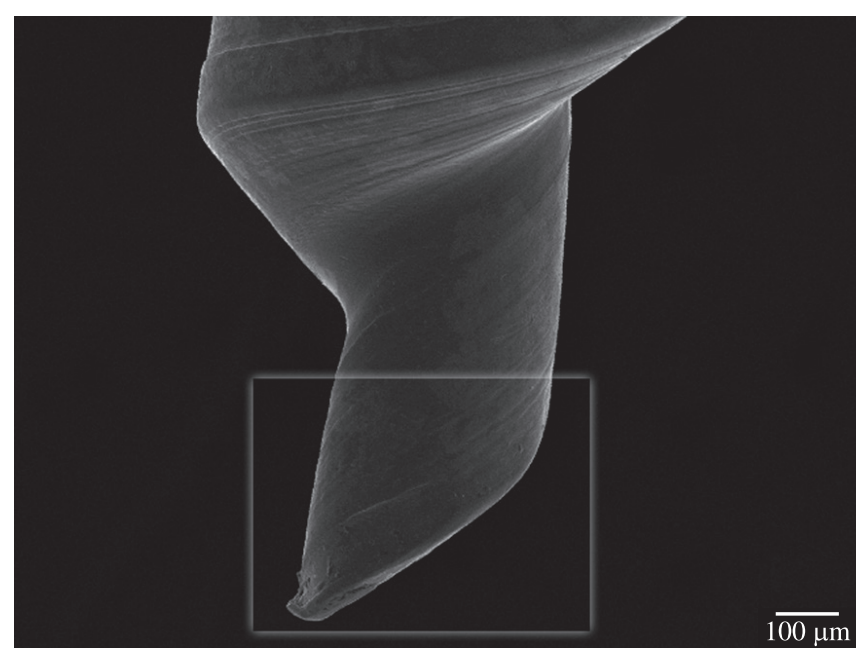

Figure 2. SEM photomicrograph of the surface morphology of the tip of a retrieved mini-implant (used for 12 months). Original magnification at $100 \times$. The highlighted portion is the part comparable to the mini-implants in Figure 3. 


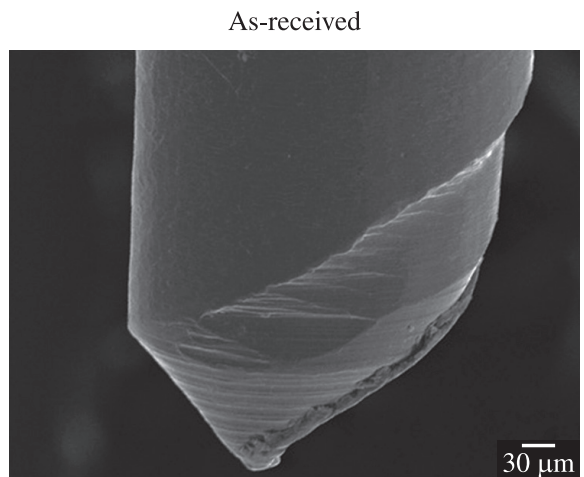

(a)

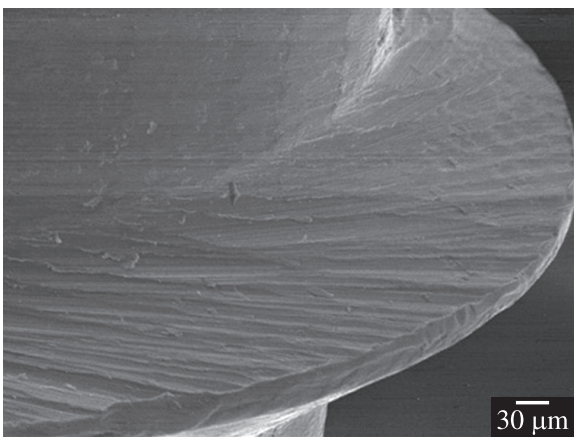

(d)

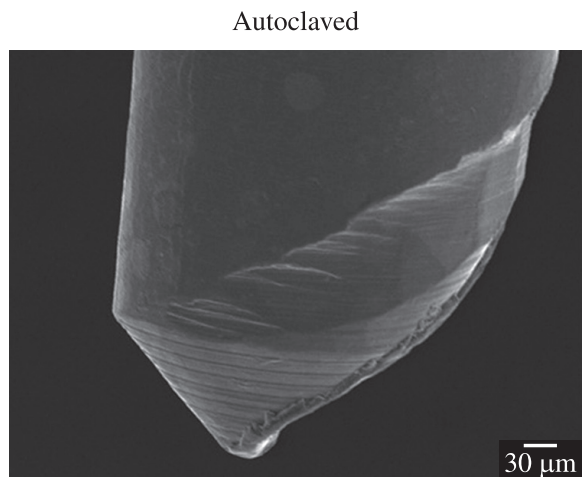

(b)

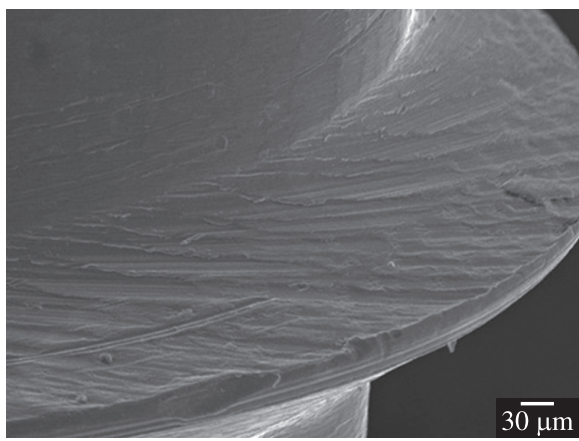

(e)

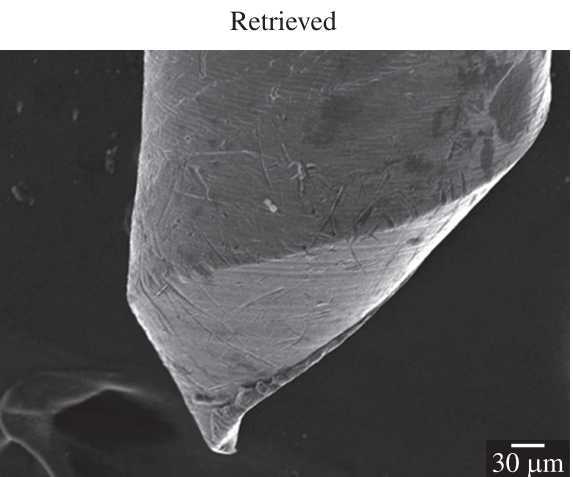

(c)

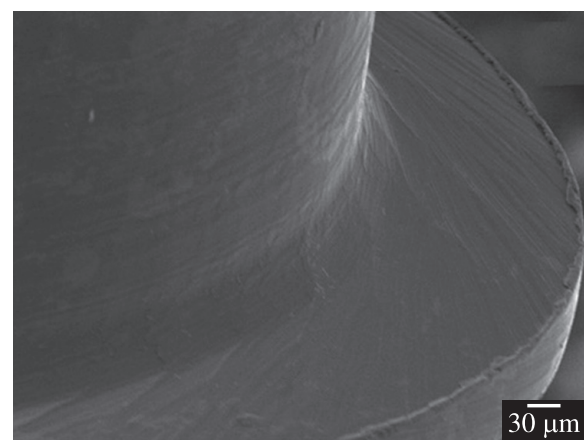

(f)

Figure 3. SEM photomicrographs of the surface morphology of mini-implants used in the present study: a) tip of as-received mini-implant; b) tip of autoclaved mini-implant; c) tip of retrieved mini-implant (used for 11 months); d) thread of as-received mini-implant; e) thread of autoclaved mini-implant; and f) thread of retrieved mini-implant (used for 11 months). Original magnification was at $250 \times$.

Descriptive statistics for the fracture torque test are described in Table 2. Comparison between groups highlighted a statistical significant difference in the fracture torque only between the asreceived and the retrieved groups (Table 3). A comparison of all groups is shown in Figure 4. The group of retrieved mini-implants showed the highest range of torque values.

\section{Discussion}

Many times during the orthodontic treatment, when a miniimplant fails, the reinstallation of a new one may be required, either in the same area after 4 to 6 weeks or in an adjacent area immediately ${ }^{14}$. Relocation of mini-implants to a better position may also be necessary in other clinical situations ${ }^{15}$, like when their function is limited by the proximity with a root and their use is still required in orthodontic mechanics. The insertion of a new mini-implant causes little discomfort and is usually well accepted by patients ${ }^{16}$.

There are ethical considerations about the re-use of invasive medical devices in different patients despite cost benefits, but they may be used again in the same patient ${ }^{17}$ if their structural integrity and mechanical properties are not altered after their prior use and sterilization. That means mini-implants could be re-used in the same patient if their properties prove to remain unaltered.

Additionally, it is important to test the effects of autoclave sterilization on mini-implants because they may be contaminated before its insertion in the bon $\mathrm{e}^{18}$ and, in this case, should be put apart to be sterilized and used in another opportunity. Besides that, if it can be proved that mini-implants could be re-used, retrieved mini-implants to be re-used should be firstly mechanically cleaned and sterilized.

This study examined as-received, autoclaved and retrieved miniimplants, comparing their surface topography by SEM analysis and resistance by a fracture torque test.

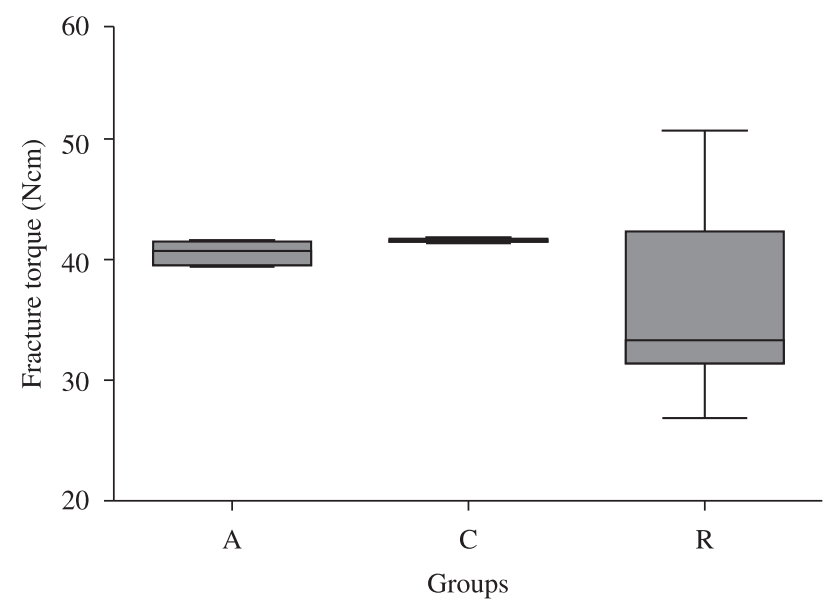

Figure 4. Box-plot of fracture torque values for all groups.

Lee and Chang ${ }^{19}$ found increased pitting and corrosion on autoclaved nickel-titanium alloy wires. Pernier et al..$^{20}$, on the other hand, observed no variation in the surface parameters after sterilization of nickel-titanium and titanium-molybdenum alloy wires. SEM analysis in this study indicates that the process of autoclave sterilization does not alter the Ti6Al4V mini-implant surface topography, as no defects in the form of pores or cracks and no image suggestive of corrosion could be identified in the autoclaved mini-implants when compared to the as-received ones.

Eliades et al. ${ }^{12}$ found morphologic and surface structural alterations in retrieved mini-implants, but no material structural changes in the form of defects or pores were documented. In this study, as well, no defects in the form of pores or cracks neither any 
Table 2. Fracture torque value $(\mathrm{Ncm})$.

\begin{tabular}{cccc}
\hline Group & Mean & SD & Range \\
\hline A & 41.00 & 0.95 & $39.60-42.10$ \\
C & 42.00 & 0.71 & $41.00-43.00$ \\
R & 36.82 & 7.41 & $26.70-51.50$ \\
\hline
\end{tabular}

Table 3. Tukey HSD post hoc test of fracture torque values. P-value

\begin{tabular}{cccc}
\hline & $\mathrm{A}$ & $\mathrm{C}$ & $\mathrm{R}$ \\
\hline $\mathrm{A}$ & - & 0.901 & 0.117 \\
$\mathrm{C}$ & 0.901 & - & $0.041^{*}$ \\
$\mathrm{R}$ & 0.117 & $0.041^{*}$ & - \\
\hline
\end{tabular}

*Statistically significant $(\mathrm{P}<0.05)$.

image suggestive of corrosion of such kind could be visualized in the retrieved mini-implants. However, their surface was smoother, six mini-implants had their tip less sharp and four had scratch marks when compared to as-received mini-implants, suggesting that its insertion and removal may have worn them. As the mini-implant studied was from the self-drilling type, the alteration in its tip may alter its properties, requiring previous bone drilling if it is to be reused. Schwartz et al. ${ }^{8}$ also observed deep scratch marks on the surface of used coverscrews.

It is also important to consider that changes in surface morphology due to cleaning and/or mechanical damage during placement and removal can result in marked changes in osteoblastic growth and differentiation ${ }^{8}$. Additionally, cell attachment levels may be lower and cell spreading reduced in titanium autoclaved surfaces ${ }^{21}$. Therefore, re-use may not be considered for mini-implants that rely on osseointegration to assure its stability, but only for those which are designed to be stabilized by mechanical interdigitation to the bone.

As to the fracture torque test, its importance is related to the knowledge of the structural integrity of the mini-implant tested and it is an important parameter to be assessed as the rotational forces associated with clinical placement and removal can cause miniimplant failure $\mathrm{s}^{15,22-23}$.

As there was no statistically significant difference between the as-received and the autoclaved groups in the fracture torque test in this study, no clinically significant changes in the resistance to fracture of autoclaved mini-implants are expected. There was, however, a statistically significant difference between the as-received $(42.00 \pm 0.71 \mathrm{Ncm})$ and the retrieved $(36.82 \pm 7.41 \mathrm{Ncm})$ groups. Even though there was this statistically significant difference, both groups showed fracture torque values beyond the insertion torque recommended by Motoyoshi et al. ${ }^{24}$, which is from 5 to $10 \mathrm{~N}$, and the removal torque observed by Chen et al. ${ }^{2}$, which ranged from 10.78 to $21.07 \mathrm{Ncm}$. The difference between retrieved mini-implants and as-received ones is relevant not only because of the mean fracture torque values they presented, but mostly because of their great range, as can be observed in Figure 4, and superficial changes observed by SEM (Figures 2 and 3). Although all fracture torque values of all mini-implants tested are above the insertion torque recommended ${ }^{24}$, the great difference between minimum and maximum torque values for the retrieved group indicates that some variables can influence or alter the resistance of used mini-implants to fracture.

It is possible that the insertion and the removal torque applied on the mini-implant prior use in the patient be responsible for such alterations in its resistance to fracture. If the mini-implant encounters extreme resistance during insertion due to high bone consistency or thick cortical bone layer, pilot drilling may be required even for self- drilling mini-implants ${ }^{5,15,25-26}$. If pilot drilling is not performed, the risk of failure by fracture of the mini-implant increases, and if it does not break, its re-use may be compromised by its possible decreased fracture torque resistance. Partial integration may also increase the removal torque of mini-implants ${ }^{15,27}$, leading to the same problem. This hypothesis should then be tested in future studies.

Based on this study, therefore, the re-use of retrieved miniimplants is not recommended, as the variables that may influence their resistance to fracture are not yet elucidated and the patient should not be submitted to the risks inherent to that procedure while this subject is not thoroughly studied.

On the other hand, autoclave sterilization of the mini-implant used in this study caused no relevant alteration on its surface morphology and fracture torque resistance when compared to an as-received miniimplant and could then be recommended clinically.

\section{Conclusion}

Retrieved mini-implants showed altered surface characteristics and a wider range of fracture torque values. Re-use of mini-implants should not be recommended, as there is not enough evidence of variables that can affect their expected resistance to fracture. Asreceived mini-implants submitted to autoclave sterilization, however, can be recommended to be used clinically.

\section{Acknowledgements}

The authors would like to thank the financial support provided by FAPERJ (Fundação de Apoio à Pesquisa do Estado do Rio de Janeiro).

\section{References}

1. Cheng S-J, Tseng I-Y, Lee J-J and Kok S-H. A prospective study of the risk factors associated with failure of mini-implants used for orthodontic anchorage. The International Journal of Oral \& Maxillofacial Implants. 2004; 19(1):100-106.

2. Chen Y-J, Chen Y-H, Lin L-D and Yao C-CJ. Removal torque of miniscrews used for orthodontic anchorage - a preliminary report. The International Journal of Oral \& Maxillofacial Implants. 2006; 21(2):283-289.

3. Papadopoulos MA and Tarawneh F. The use of miniscrew implants for temporary skeletal anchorage in orthodontics: a comprehensive review. Oral Surgery, Oral Medicine, Oral Pathology, Oral Radiology, and Endodontics. 2007; 103(5):e6-e15.

4. Wiechmann D, Meyer U and Büchter A. Success rate of mini- and microimplants used for orthodontic anchorage: a prospective clinical study. Clinical Oral Implants Research. 2007; 18(2):263-267.

5. Iijima M, Muguruma T, Brantley W, Okayama M, Yuasa T and Mizoguchi I. Torsional properties and microstructures of miniscrew implants. American Journal of Orthodontics and Dentofacial Orthopedics. 2008; 134(3):333.e1-333.e6.

6. Lim S-A, Cha J-Y and Hwang C-J. Insertion torque of orthodontic miniscrews according to changes in shape, diameter and length. The Angle Orthodontist. 2008; 78(2):234-240.

7. Reynders R, Ronchi L and Bipat S. Mini-implants in orthodontics: a systematic review of the literature. American Journal of Orthodontics and Dentofacial Orthopedics. 2009; 135(5):564.e1-564.e19.

8. Schwartz Z, Lohmann CH, Blau G, Blanchard CR, Soskolne AW, Liu Y et al. Re-use of implant coverscrews changes their surface properties but not clinical outcome. Clinical Oral Implants Research. 2000; 11(3):183-194.

9. Sonis AL. Air abrasion of failed bonded metal brackets: a study of shear bond strength and surface characteristics as determined by scanning electron microscopy. American Journal of Orthodontics and Dentofacial Orthopedics. 1996; 110(1):96-98.

10. Pringle RA, Leman RB, Kratz JM and Gillette PC. An argument for pacemaker reuse: pacemaker mortality in 169 patients over ten years. Pacing and Clinical Electrophysiology. 1986; 9(6 Pt2):1295-1298. 
11. Grimandi G, Sellal O, Grimandi F and Crochet D. Risks of reusing coronary angioplasty catheters: results of an experimental study. Catheterization and Cardiovascular Diagnosis. 1996(2); 38:123-130.

12. Eliades T, Zinelis S, Papadopoulos MA and Eliades G. Characterization of retrieved orthodontic miniscrew implants. American Journal of Orthodontics and Dentofacial Orthopedics. 2009; 135(1):10.e1-10.e7.

13. Pithon M, Nojima L, Nojima M and Ruellas AC. Comparative study of fracture torque for orthodontic mini-implants of different trademarks. Oral Surgery. 2008; 1(2):84-87.

14. Baek S-H, Kim B-M, Kyung S-H, Lim JK and Kim YH. Success rate and risk factors associated with mini-implants reinstalled in the maxilla. The Angle Orthodontist. 2008; 78(5):895-901.

15. Carano A, Velo S, Leone P and Siciliani G. Clinical applications of the miniscrew anchorage system. Journal of Clinical Orthodontics. 2005; 39(1):9-24.

16. Chen Y-J, Chang H-H, Huang C-Y, Hung H-C, Lai EH-H and Yao C-CJ. A retrospective analysis of the failure rate of three different orthodontic skeletal anchorage systems. Clinical Oral Implants Research. 2007; 18(6):768-775

17. Karch M, Alt E, Schmitt C and Schömig A. Reimplantation of an infected, abdominally implanted defibrillator in the subpectoral region. The Journal of Cardiovascular Surgery. 1996; 37(1):67-70.

18. Dorst J. Sterilization requirements for miniscrew placement. American Journal of Orthodontics and Dentofacial Orthopedics. 2009; 136(5):621-622.

19. Lee SH and Chang YII. Effects of recycling on the mechanical properties and the surface topography of nickel-titanium alloy wires
American Journal of Orthodontics and Dentofacial Orthopedics. 2001; 120(6):654-663.

20. Pernier C, Grosgogeat B, Ponsonnet L, Benay G and Lissac M. Influence of autoclave sterilization on the surface parameters and mechanical properties of six orthodontic wires. European Journal of Orthodontics. 2005; 27(1):72-81.

21. Vezeau PJ, Koorbusch GF, Draughn RA and Keller JC. Effects of multiple sterilization on surface characteristics and in vitro biologic responses to titanium. Journal of Oral and Maxillofacial Surgery. 1996; 54(6):738-746.

22. Adelson RT, DeFatta RJ and Ducic Y. Integrity of craniofacial plating systems after multiple sterilization procedures. Journal of Oral and Maxillofacial Surgery. 2007; 65(5):940-944.

23. Morais LS, Serra GG, Palermo EFA, Andrade LR, Müller CA, Meyers MA et al. Systemic levels of metallic ions released from orthodontic mini-implants. American Journal of Orthodontics and Dentofacial Orthopedics. 2009; 135(4):522-529.

24. Motoyoshi M, Hirabayahi M, Uemura M and Shimizu N. Recommended placement torque when tightening an orthodontic mini-implant. Clinical Oral Implants Research. 2006; 17(1):109-114.

25. Carano A, Lonardo P, Velo S and Incorvati C. Mechanical properties of three different commercially available miniscrews for skeletal anchorage. Progress in Orthodontics. 2005; 6(1):82-97.

26. Jolley TH and Chung C-H. Peak torque values at fracture of orthodontic miniscrews. Journal of Clinical Orthodontics. 2007; 41(6):326-328.

27. Park H-S, Jeong S-H and Kwon O-W. Factors affecting the clinical success of screw implants used as orthodontic anchorage. American Journal of Orthodontics and Dentofacial Orthopedics. 2006; 130(1):18-25. 